\title{
Heat Generation during the Fatigue of a Cellular Al Alloy
}

\author{
A. RABIEI, A.G. EVANS, and J.W. HUTCHINSON
}

\begin{abstract}
The heat generation from a notch during the compression-compression fatigue of a cellular Al alloy has been measured and compared with a model. The measurements indicate that heat is generated because of hysteresis occurring in narrow cyclic plastic zones outside the notch. This process continues until the notch closes. At closure, a brief period of heat generation arises because of friction along the notch faces. A plasticity model based on the Dugdale zone is shown to provide a reasonably accurate characterization of the heat generated, with the proviso that an "ineffective" zone be transposed onto the notch tip. It is found that the temperatures generated are too small to cause fatigue by thermal softening. A fatigue mechanism based on either geometric softening of the cells or crack growth in the cell walls is implied.
\end{abstract}

\section{INTRODUCTION}

CELlULAR metals have been shown to experience fatigue degradation in both tension and compression. ${ }^{[1,2]}$ This degradation is manifested as a rapid increase in strain after a quiescent nucleation period wherein strain accumulation occurs slowly and stably (Figure 1(a)). The consequence is an endurance limit occurring at some fraction of the monotonic flow strength. This limit may be used for design purposes. $^{[2]}$ As fatigue progresses, the effective Young's modulus decreases (Figure 1(b)), indicative of damage mechanisms operating in the material. ${ }^{[2]}$ The abrupt increase in strain beyond the nucleation stage occurs in discrete bands which have a thickness equal to about one cell size. The mechanisms responsible for localizing the strains into bands and limiting life have received minimal attention. Optical observations have indicated that the cell walls encompassing some of the relatively large cells are susceptible to plastic buckling at the peak stress ${ }^{[1]}$ (Figure 2). These buckled walls experience larger-than-average cyclic strains and submit to a cyclic softening mechanism that induces and spreads a lateral degradation zone, which eventually collapses the material along an entire band.

The exaggerated cyclic plastic strains implied by these observations suggest that heat is generated locally around strain concentrators and that the thermal flux be a measure of the plastic work accompanying each strain cycle. The present article provides a demonstration of this local heating effect and connects the flux to the plastic deformation. It also addresses the possibility that fatigue is governed by thermal softening as a result of the heat generated.

Heat generation during crack growth and fatigue has previously been studied in the following two situations. ${ }^{[3-6]}$ (1) In metals having low thermal conductivity, particularly amorphous Ti alloys, appreciable heat is generated during rapid crack extension, with local elevations in temperature around the crack reaching about $20^{\circ} \mathrm{C}^{[3,4]}(2)$ In polymers subject to generalized cyclic straining, appreciable heating has been measured, with temperature elevations in PTFE

A. RABIEI, Postdoctoral Fellow, and J.W. HUTCHINSON, Professor, are with the Division of Engineering and Applied Sciences, Harvard University, Cambridge, MA 02138. A.G. EVANS, Professor and Director, is with the Materials Institute, Princeton University, Princeton, NJ 08540.

Manuscript submitted May 7, 1999. exceeding $100{ }^{\circ} \mathrm{C}$ at frequencies in the $30 \mathrm{~Hz}$ range, again because of low its thermal conductivity. ${ }^{[5,6]}$ To the authors' knowledge, there have been no studies either in metals or polymers of heat generated locally around stationary cracks subject to cyclic loading.

\section{EXPERIMENTAL METHODS}

The thermal measurements are performed on a well-characterized, commercially available, closed-cell $\mathrm{Al}$ alloy, having the trade name ALPORAS. ${ }^{[1,7-9]}$ Rectangular compression specimens were cut from castings of this material by electrodischarge machining (EDM), as described elsewhere ${ }^{[7]}$ Most tests were performed on samples with dimensions of $65 \times 55 \times 55 \mathrm{~mm}$. A few tests were conducted on narrower samples, 12-mm thick. A small elliptical hole $(12 \times 4 \mathrm{~mm})$ was introduced into the center section by EDM. The minor axis of the hole was slightly larger than the largest cells. ${ }^{[1]}$

Accordingly, the hole provides a well-defined strain concentrator that should localize the onset of fatigue degradation.

For testing, both ends of the specimen were thermally insulated. This was achieved using inserts between the specimen and the loading platens. This insulating layer was needed to prevent heat generated by friction at the platens, as well as by the cyclic operation of the testing machine, from diffusing into the test specimen.

Testing was performed in a compression-compression mode, using a servohydraulic machine subject to an $R$ ratio of 0.1 at a frequency $\lambda$ of $10 \mathrm{~Hz} .^{[1,2]}$ The maximum stress was varied between 0.8 and $0.95 \sigma_{0}$, where $\sigma_{0}$ is the monotonic plateau stress. ${ }^{[1,7,9]}$ Strains were measured using a linear variable displacement transducer and were recorded digitally. Images obtained by optical microscopy and scanning electron microscopy were taken during testing to characterize the deformation phenomena.

Thermal imaging of one of the side surfaces was performed using a high-resolution infrared (IR) camera (Amber-Galileo) with a $256 \times 256$ array and a $30 \mu \mathrm{m}$ pixel size. A $50 \mathrm{~mm}$ IR lens was used to provide a magnification of $0.215 \mathrm{~mm} / \mathrm{pixel}$. The surface of the specimen was covered with a black thin film to control its emissivity. In order to monitor small $\left(<0.1{ }^{\circ} \mathrm{C}\right)$ variations in temperature, an integration time of $1.2 \mathrm{~ms}$ was selected, with 15 images 


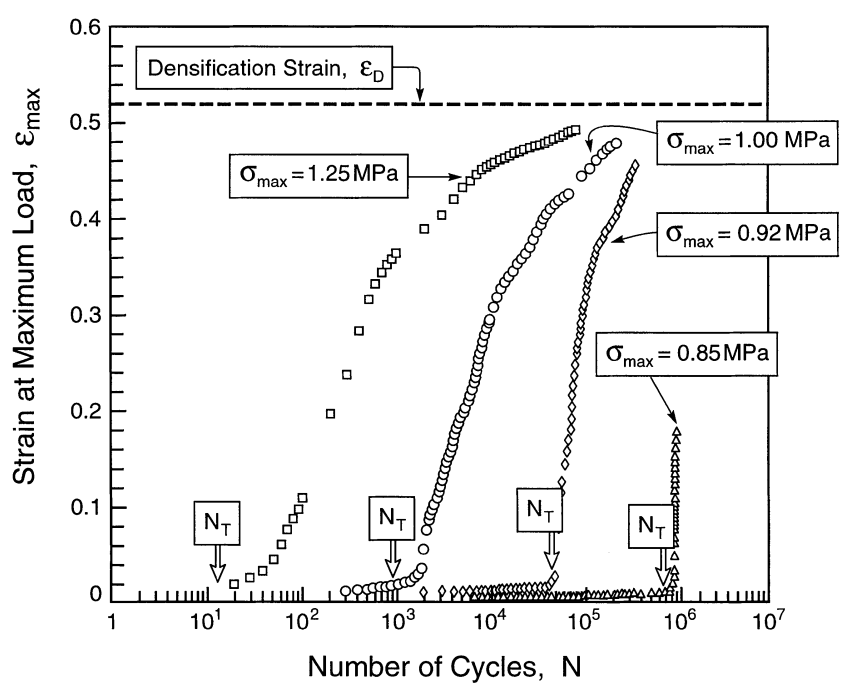

(a)

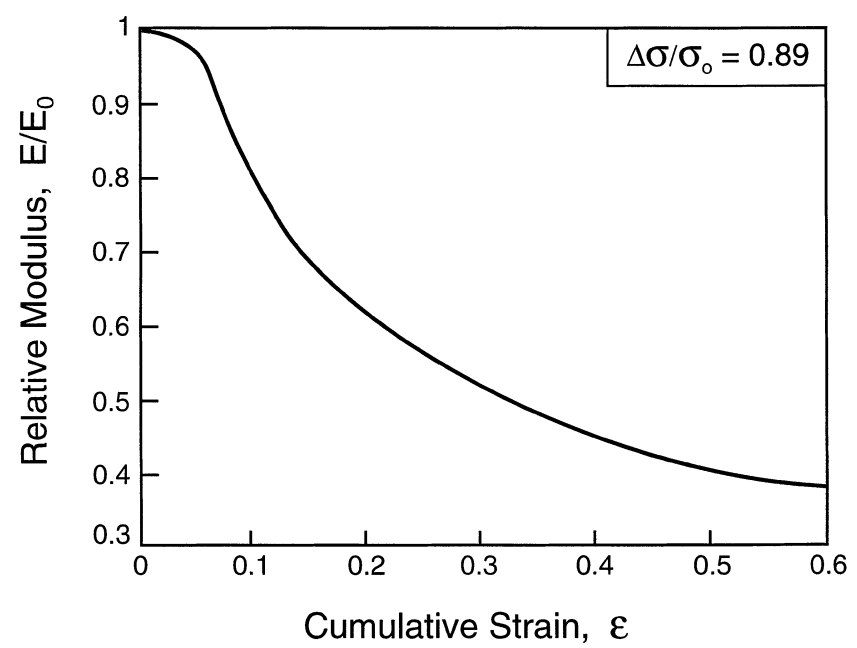

(b)

Fig. 1-(a) The cumulative strain at maximum stress plotted as a function of the number of cycles for the closed cell Al, Alporas. (b) The degradation in compression modulus that occurs as strain accumulates.

acquired at a rate of 60 frames/s, by employing a digital control system. The temperature was calibrated by replacing the specimen with a black body, which was gradually heated, and the actual temperature was monitored by using thermocouples. Comparing the output of the camera with that of the thermocouples related the digital counts to the temperature.

\section{MEASUREMENTS AND OBSERVATIONS}

The cumulative strains $\left(\varepsilon_{\max }(N)\right)$ exhibited by the specimens with elliptical holes are summarized in Figure 3, using the net-section stress as the load reference. The strain is that remote from the hole. Note that the curves have a form similar to that found for an un-notched material, ${ }^{[1,2]}$ including a quiescent phase followed by rapid strain accumulation beyond a critical number of cycles $\left(N_{T}\right)$ and eventual saturation.

It is difficult to compare $\varepsilon_{\max }(N)$ curves for specimens with and without holes, because the strains are measured on a different basis. However, it is reasonable to compare trends

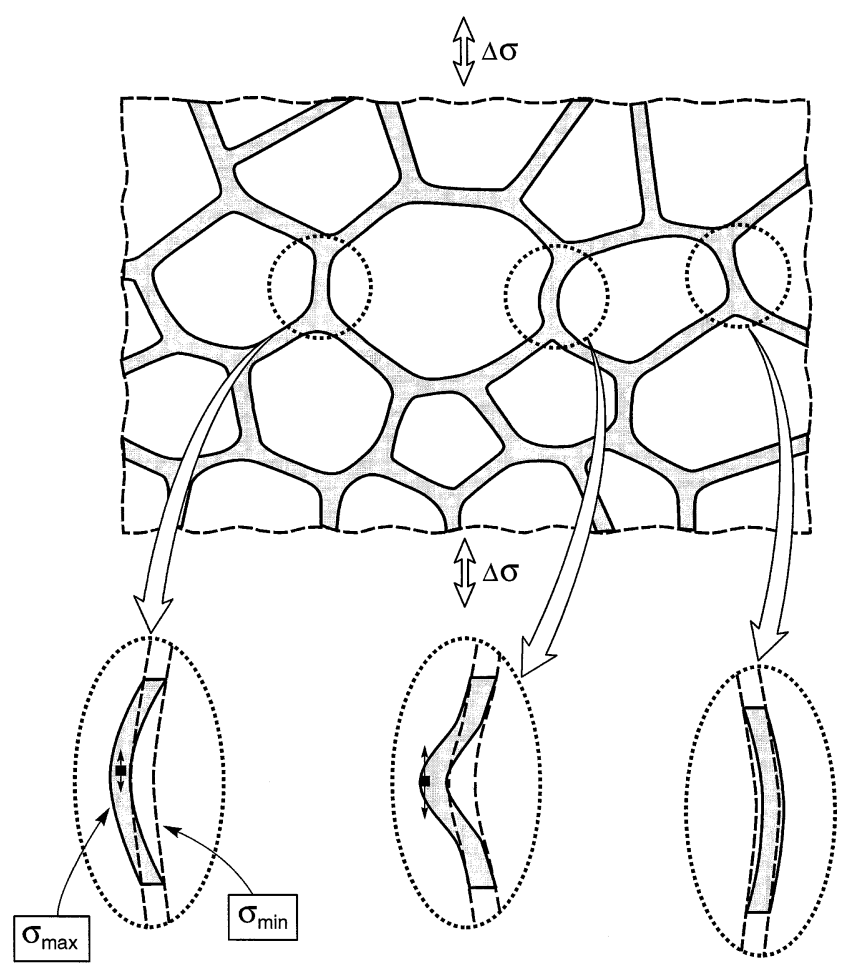

Fig. 2-A schematic indicating the plastic buckling of cell walls that occurs at the early stages of fatigue in compression.



Fig. 3-Cumulative remote strain measured at peak load as a function of the number of cycles for three different values of the maximum net section stress, $\sigma_{\text {net }}$, compared with some results from Fig. 1(a) at a similar maximum stress level, $\sigma_{\max }$.

in $N_{T}$ with the net-section stress (Figure 4). This comparison reveals moderate notch strengthening. That is, rapid strain accumulation occurs after a somewhat larger number of cycles in the presence of a hole, at the equivalent net-section stress. Analogous (small) strengthening effects have been reported previously upon both monotonic and cyclic loading. ${ }^{[2,7]}$

Subsequent to the jump in strain at $N_{T}$, optical images reveal that the hole systematically closes during the period of rapid strain accumulation and is fully closed at the saturation stress. This effect is most vividly demonstrated in the thermal images described subsequently. As the hole closes, a collapse 


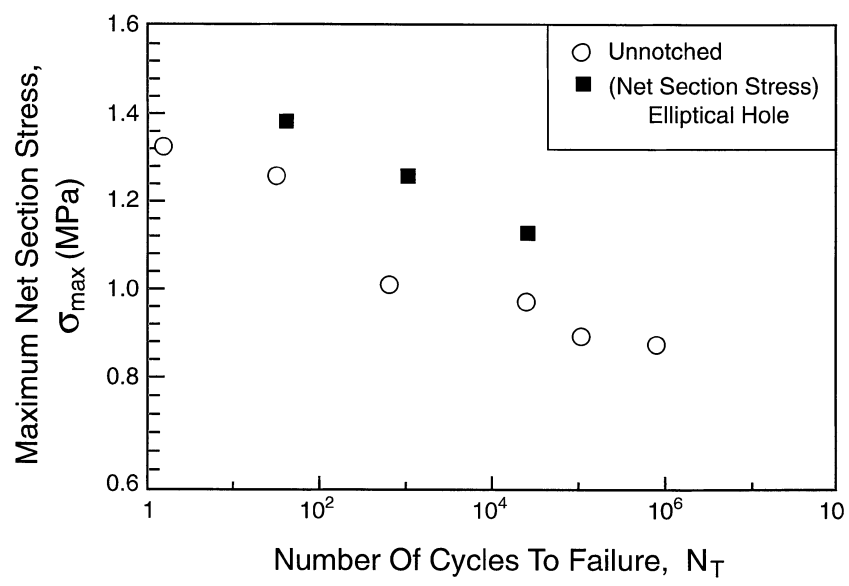

Fig. 4-Effect of net section stress on the cycles to "failure," $N_{T}$, compared with results for un-notched material.
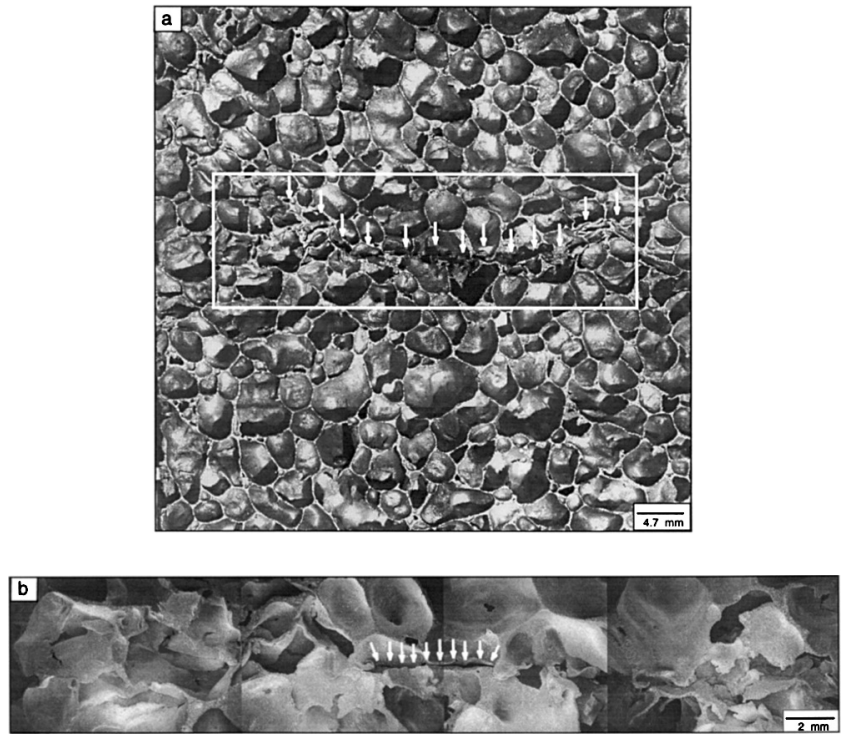

Fig. 5-Images of the region around the hole taken after the hole has collapsed. (a) General optical image: the collapse band is identified with arrows. (b) Scanning electron microscope image showing the closed hole (arrows) and damaged cells within the collapse band.

band forms along the plane containing the hole (Figure 5). These measurements and observations affirm that fatigue nucleates preferentially at strain concentrators, yet their influence on fatigue life is small.

A sequence of thermal images taken at a peak stress of $0.95 \sigma_{0}$ (Figure 6) illustrates the nature of the heating effect as well as the simultaneous collapse of the elliptical hole. Similar effects occur at other stress levels. It is apparent that, while the hole is still present, heat is generated from a small, elongated region just outside the major axes of the ellipse and diffuses out as cycling proceeds. After the hole has collapsed, a zone of additional heat generation arises along the contacting faces, resulting in a hot zone with elliptical symmetry (Figure 6, image 7). This mode of heating has a relatively short duration, stopping when strain saturation is complete, whereupon the temperature redistributes and then begins to cool by thermal diffusion (image 8).

The time variation of the temperature at a site near the hole that experiences the largest rise is plotted in Figure $7(a)$. Note that the temperature at this site continues to rise during the strain-accumulation phase, as the hole is closing (Figure 7(a), images 1 through 6). The temperature changes from $23.7^{\circ} \mathrm{C}$ at the beginning to $24.3{ }^{\circ} \mathrm{C}$ at the peak. The cooling that occurs after hole closure is plotted in Figure 7(b). The distribution of temperature along $x$ and $y$ at several times prior to closure is plotted in Figure 8 (images 1, 2, 4, 6 , and 8 ). These temperatures will be compared with the model described next. Note that the predicted and measured temperatures are both for the solid material in the cell walls.

\section{ANALYSIS}

The temperature history is modeled with the objective of relating the heat-generation rate at the source to the cyclic plasticity. The analysis addresses heat generation before closure. The subsequent heating, caused by cyclic contact of the surfaces of the closed hole, is not examined. Initially, the heat sources are considered to comprise two thin, cyclic plastic zones at the major axis of the elliptical hole. Plasticity characteristics analogous to those for a Dugdale zone have been assumed, because this cellular $\mathrm{Al}$ strains uniaxially (zero plastic Poisson ratio). ${ }^{[10]}$ The material within the Dugdale zone can undergo plastic deformation without straining in the thickness direction. The ellipse is modeled as a slit, or open crack. The surfaces of the crack are assumed to be insulating, and, thus, the thermal problem has a symmetry plane along the center across which no heat flows.

The model problem is depicted in Figure 9. It comprises two active zones of cyclic plasticity, having a length of $s$, outside an elliptical hole having a major axis of 2a. It is subject to a cyclic stress $\left(\Delta \sigma_{\infty}\right)$ with $R>0$. In each cycle, elements of material within the active zone undergo plastic displacements, with a magnitude of $\delta$, at a flow stress equal to the plateau strength for the material. Accordingly, the hysteresis loop has a magnitude of $2 \sigma_{0}$ and a width of $\delta$ (Figure 9), such that the plastic work per cycle within a unit thickness, per unit length of plastic zone, is

$$
W_{p 1}=2 \sigma_{0} \delta
$$

The solution to the cyclic Dugdale model gives

$$
\delta=\frac{2 \sigma_{0} a}{E} \bar{\delta}(\bar{s}, \bar{x})
$$

where

$$
\begin{aligned}
& \bar{s} \equiv s / a=\sec \left(\frac{\pi \Delta \sigma_{\infty}}{4 \sigma_{0}}\right)-1 \\
& \bar{x}=x / a
\end{aligned}
$$

with $E$ being the Young's modulus and $x$ the distance from the center of the ellipse (Figure 9). The quantity $\bar{\delta}$ is given by $(1<\bar{x} \overline{<} 1+\bar{s})$

$$
\begin{aligned}
(\pi / 8) \bar{\delta}(\bar{s}, \bar{x})= & \left(\sin ^{-1}\left(\frac{1}{1+\bar{s}}\right) \sqrt{(1+\bar{s})^{2}-\bar{x}^{2}}\right. \\
& -\bar{x} \tanh ^{-1}\left(\frac{((1+\bar{s}) / \bar{x})^{2}-1}{(1+\bar{s})^{2}-1}\right)^{1 / 2} \\
& \left.+\tanh ^{-1}\left(\frac{1-(\bar{x} /(1+\bar{s}))^{2}}{1-1 /(1+\bar{s})^{2}}\right)^{1 / 2}\right)
\end{aligned}
$$



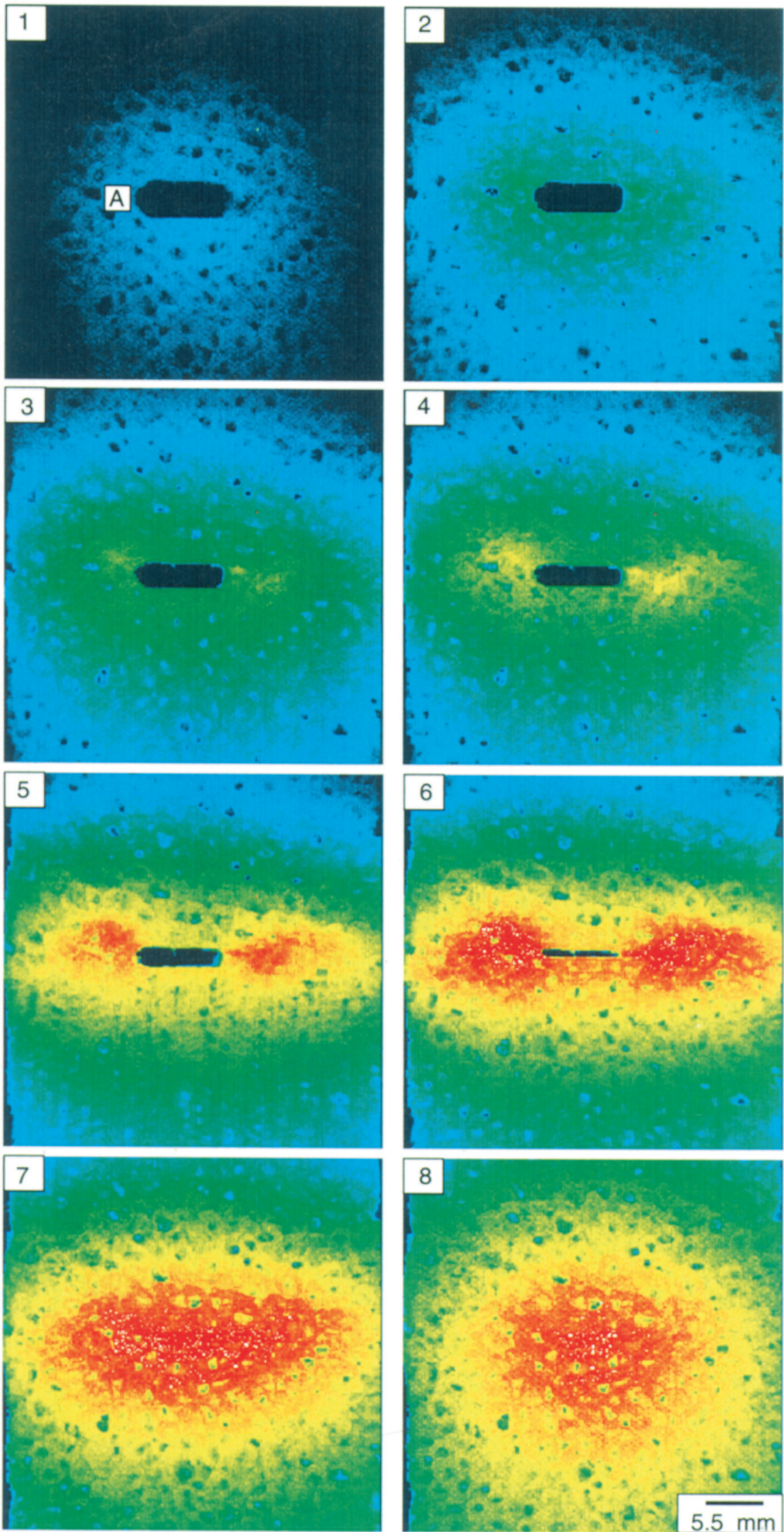

Fig. 6-A sequence of thermal images taken on the specimen tested at a net section stress of 1.4 MPa. The images are taken after the numbers of cycles indicated on Fig. 3 as (1) through (8). 


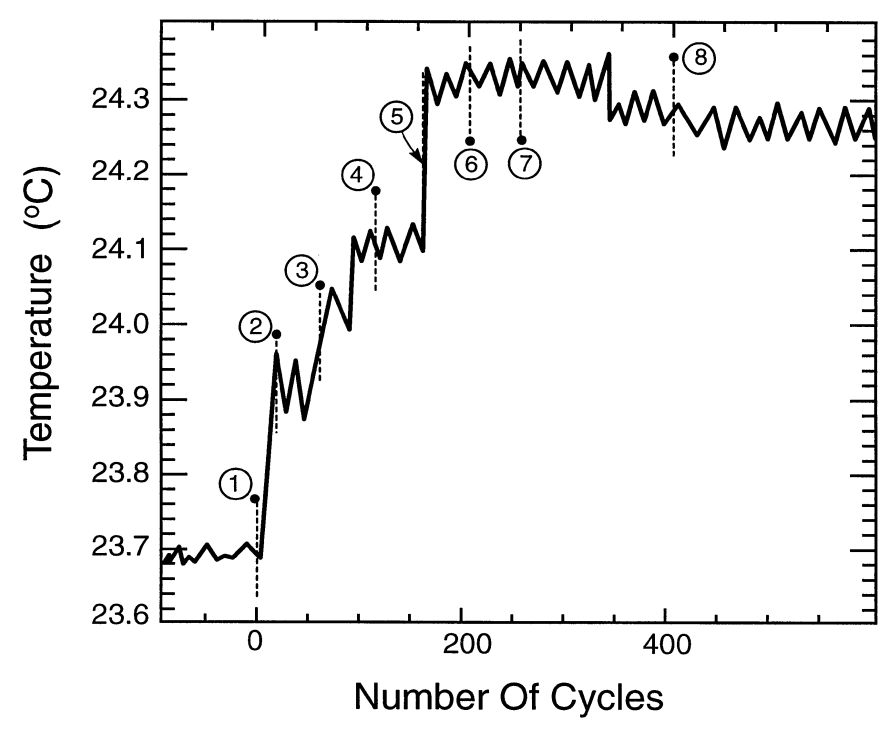

(a)

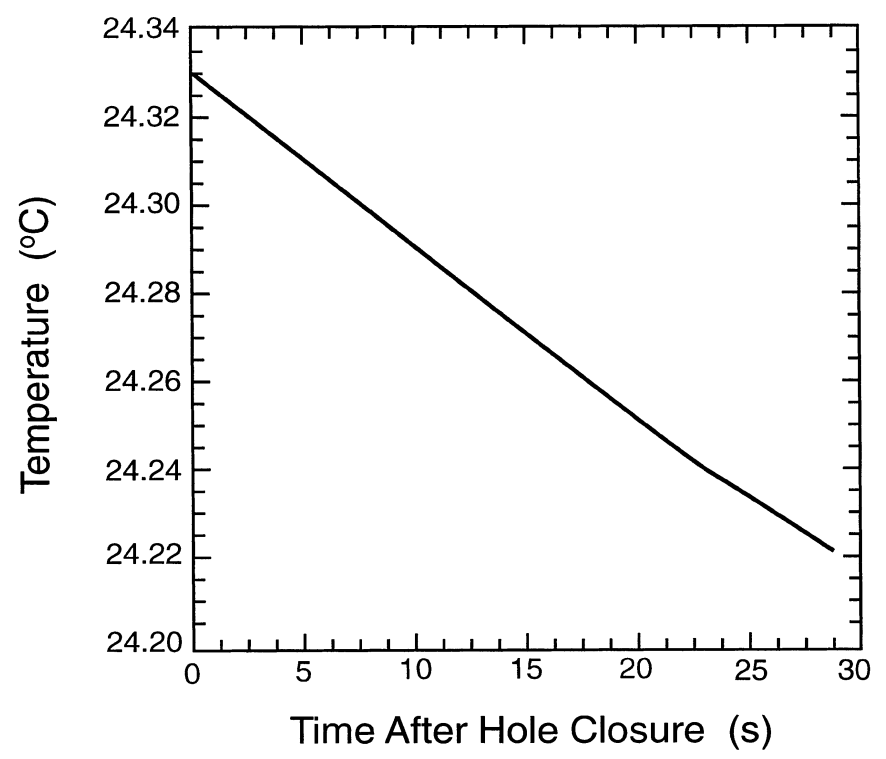

(b)

Fig. $7-(a)$ Variation in temperature with time for the same specimen as Fig. 6. The site of the measurement is just outside the major axis of the hole, within an area $150 \times 150 \mu \mathrm{m}(\bar{x}=1$ and $\bar{y}=0.1)$. (b) Cooling trend at the equivalent location after the hole has closed.

$$
-\left(\frac{\pi}{2}-\cos ^{-1}\left(\frac{1}{1+\bar{s}}\right)\right)\left((1+\bar{s})^{2}-\bar{x}^{2}\right)^{1 / 2}
$$

The cyclic results are obtained from the Dugdale model under monotonic load by replacing $\sigma_{0}$ with $2 \sigma_{0}$, as discussed in Reference 11. The expression for $\bar{\delta}$ is obtained with the aid of the fundamental solutions compiled in Reference 12.

The plastic work in the Dugdale zone is converted into heat, starting at a time of $t=0$, at a temperature of $T=$ $T_{0}$. Accordingly, the heat generated $(d q)$ per second in an element of the plastic zone $(d x)$ per unit thickness is

$$
\hat{d q} \equiv W_{p 1} \lambda d x=2 \sigma_{0} \delta(x) \lambda d x
$$

where $\lambda$ is the cycling frequency.
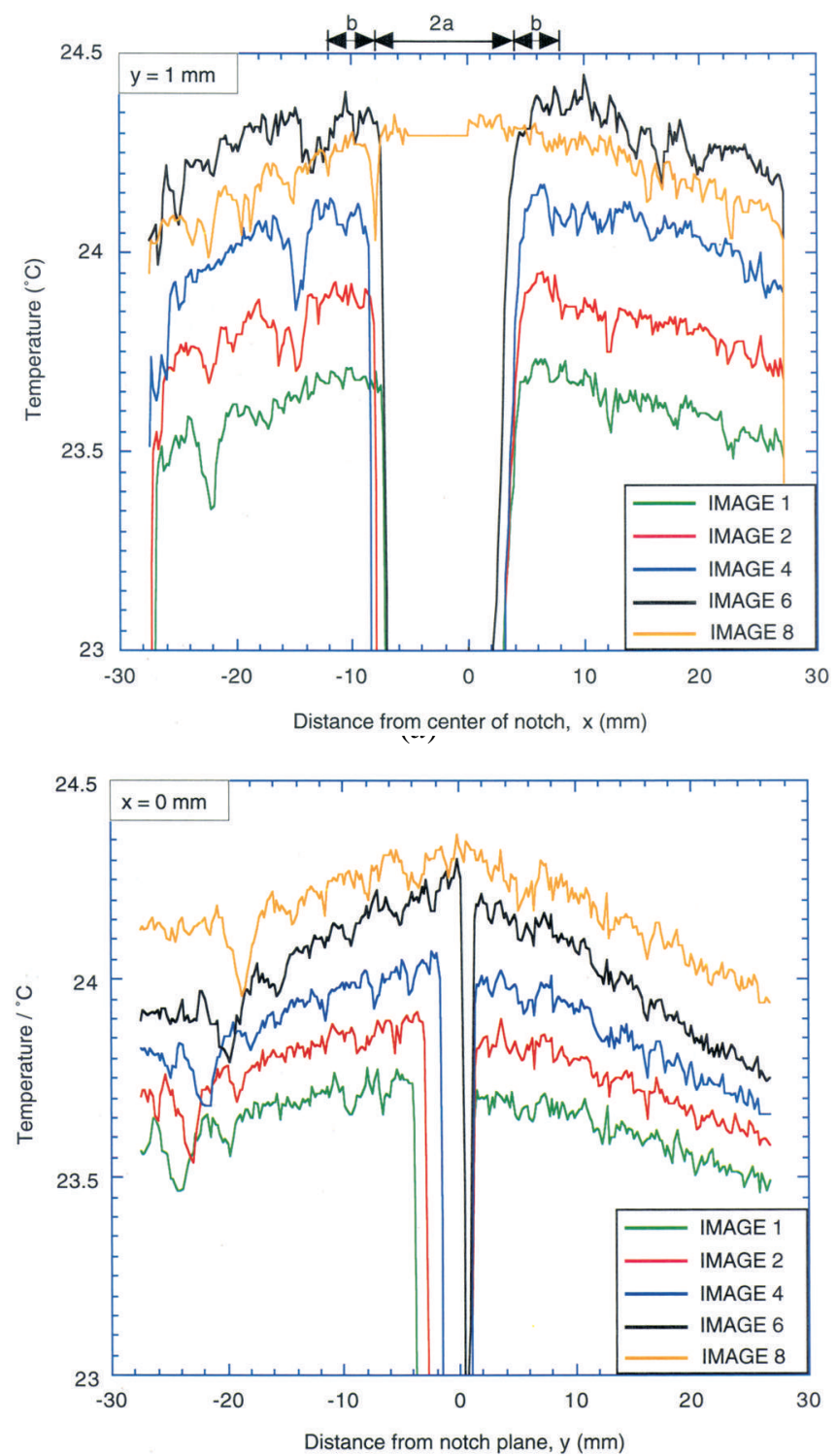

(b)

Fig. 8-Spatial variations in temperature corresponding to images $1,2,4$, 6, and 8 in Fig. 6: (a) along $x$ at $y=1 \mathrm{~mm}$ and (b) along $y$ at $x=0$ measured from the center of the hole. Note that the temperatures are not symmetric about the center of the hole, probably because of damaged material on one side.

The elevation in temperature $(\Delta T)$ caused by the heat generation can be obtained by superimposing a distribution of kernel solutions along the plastic zone. The fundamental solution corresponds to the problem of an infinite slab initially at uniform temperature, with a point source at $\left(x^{\prime}, 0\right)$ producing a uniform rate of heat per unit thickness, starting at $t=0$. With $k$ and $\kappa$ as the thermal conductivity and diffusivity, respectively, the kernel solution for the temperature change is

$$
\Delta T(x, y, t)=\frac{q}{4 \pi k} f(\xi)
$$

where 


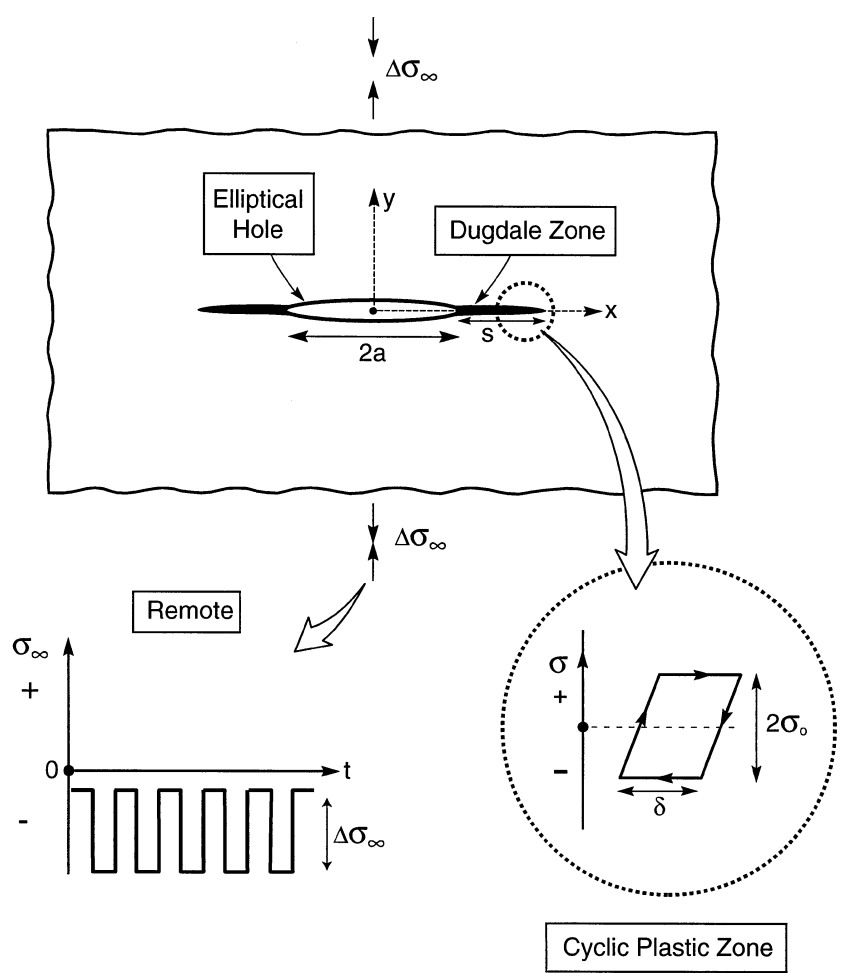

Fig. 9-A schematic showing the zones of cyclic plastic straining around the hole (referred to as a Dugdale zone). The hysteresis loop that results in heat generation per cycle is also indicated.

$$
\xi=\left(\left(x-x^{\prime}\right)^{2}+y^{2}\right) /(\kappa t)
$$

and

$$
f(\xi)=\frac{1}{4 \pi} \int_{\xi}^{\infty} \eta^{-1} e^{-\eta / 4} d \eta
$$

By superposition, the solution for the temperature change for the Dugdale model then becomes

$$
\begin{aligned}
\Delta T(x, y, t)= & (4 \lambda / k)\left(\sigma_{0}^{2} a^{2} / E\right) \int_{1}^{1+\bar{s}} \bar{\delta}\left(\bar{s}, \bar{x}^{\prime}\right)\left(f\left(\xi_{L}\right)\right. \\
& \left.+f\left(\xi_{R}\right)\right) d \bar{x}^{\prime}
\end{aligned}
$$

where

$$
\begin{gathered}
\xi_{L}=\left(\left(\bar{x}+\bar{x}^{\prime}\right)^{2}+\bar{y}^{2}\right) / \bar{t}, \\
\xi_{R}=\left(\left(\bar{x}-\bar{x}^{\prime}\right)^{2}+\bar{y}^{2}\right) / \bar{t}, \\
\bar{x}=x / a, \bar{y}=y / a
\end{gathered}
$$

and

$$
\bar{t}=t \kappa / a^{2}
$$

The nondimensional form is

$$
\begin{aligned}
\sum_{\left(\bar{x}, \bar{y}, \bar{t}, \Delta \sigma_{\infty} / \sigma_{0}\right)} & \equiv \frac{\Delta T K E}{\lambda \sigma_{0}^{2} a^{2}} \\
& =4 \int_{1}^{1+\bar{s}} \bar{\delta}\left(\bar{s}, \bar{x}^{\prime}\right)\left(f\left(\xi_{L}\right)+f\left(\xi_{R}\right)\right) d \bar{x}^{\prime}
\end{aligned}
$$

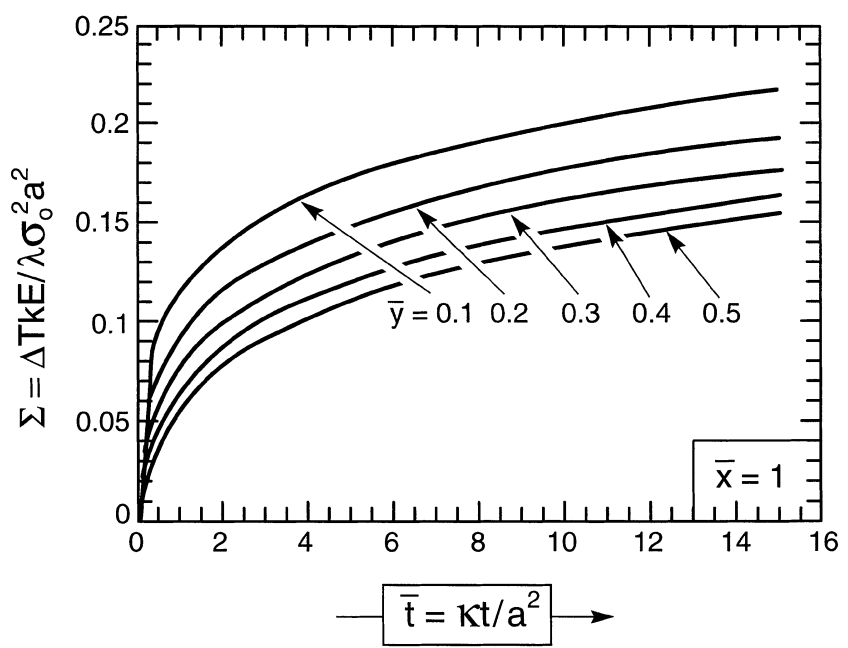

Fig. 10-Calculated variation in the nondimensional temperature, $\Sigma$, with time, $\bar{t} \equiv \kappa t / a^{2}$.

Integration of Eq. [6] gives the results plotted in Figures 10 and 11 (for $\Delta \sigma_{\infty} / \sigma_{0}=0.9$, the range used in the present experiments). The variation in the nondimensional temperature $(\Sigma)$ with time $(\bar{t})$ is indicated in Figure 10 at $\bar{x}=1$ and for various values of $\bar{y}$. The contours representing regions of constant $\Sigma$ around the holes are plotted in Figure 11. These results are used subsequently to compare with the experimental measurements.

\section{INTERPRETATION OF MEASUREMENTS}

A cursory comparison of the measured temperatures with those simulated indicates a spatial discrepancy. Namely, while the location exhibiting the largest $\Delta T$ in the simulation is at the notch tip (Figure 11), it is at some distance $(b)$ ahead of the notch in the measurements (Figures 6 and 8). If an "ineffective zone" is assumed, such that the notch tip in the simulation is relocated to $x=a+b$ (Figure 8), there appears to be spatial correspondence. The length of the ineffective zone needed to establish this coincidence $(b \approx$ $4 \mathrm{~mm}$ ) represents about one cell. It is surmised that the first row of cells was damaged when the notch was introduced, whereupon full cyclic plasticity (Figure 9) is sustained only in a zone, $x>a+b$.

The measurements are interpreted by using the thermomechanical properties of the ALPORAS material summarized in Table I. Initially, the nondimensional times are estimated by recalling that the loading frequency is $\lambda=10 \mathrm{~Hz}$. Using the literature value for the thermal diffusivity (Table I) and by using a notch length of $a+b$, the time at image 6 in Figure 6 is $\bar{t} \approx 4$. The focus of further analysis is on shorter times.

An estimate of the nondimensional temperature is made from Figure 7(a) in the time period up to image 6. For this purpose, a choice for the Young's modulus is needed, consistent with its role in governing the plastic displacement $(\delta(2))$. The degraded value $E / E_{0}=0.4$ is chosen (Figure 1(b)). Then, in conjunction with the parameters from Table I, at the peak, the nondimensional temperature reaches $\Sigma=$ 0.3 . This is about twice the level found in the simulations at the equivalent time, $\bar{t}=4$ (Figure 10). Given uncertainties 

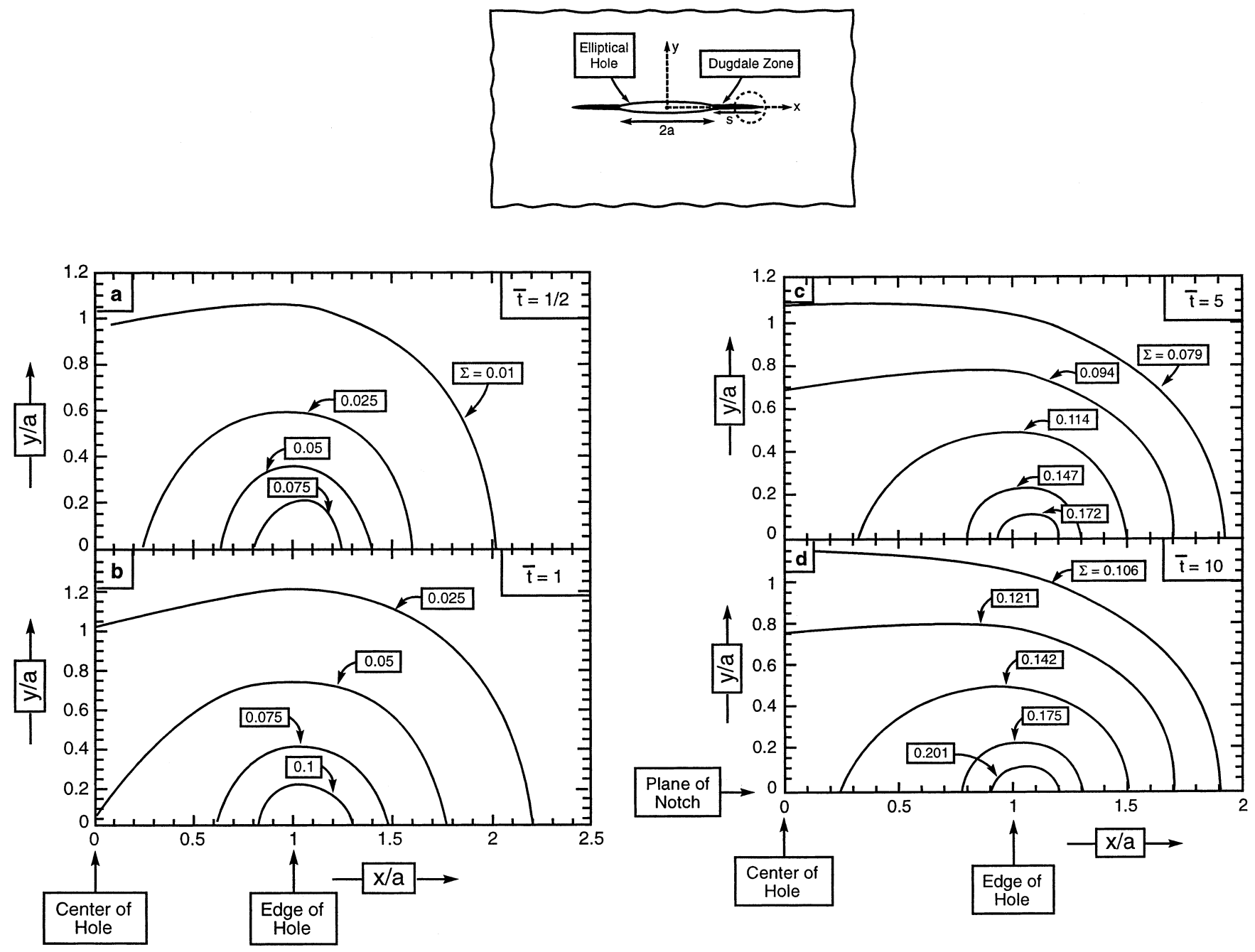

Fig. $11-$ Contours of constant temperature, $\Sigma$, around the hole plotted for four different times, $\bar{t}$, between $1 / 2$ and 10 .

Table I. Material Properties ${ }^{[13]}$

\begin{tabular}{ll}
\hline Young's modulus & $E_{0}(\mathrm{GPa})=1.1$ \\
Plateau flow strength & $\sigma_{0}(\mathrm{MPa})=1.4$ \\
Plastic Poisson ratio & $v_{p}=0$ \\
Thermal conductivity & $k(\mathrm{~W} / \mathrm{m} \mathrm{K})=2.5$ \\
Thermal diffusivity & $\kappa\left(\mathrm{m}^{2} / \mathrm{s}\right)=2 \times 10^{-5}$ \\
\hline
\end{tabular}

in some of the material properties and the approximate nature of the model, the results are considered to be sufficiently consistent with cyclic plasticity as the primary heat-generating phenomenon.

The temperatures generated are too small to cause thermal softening of the material. The fatigue effect must, therefore, be attributed either to geometric softening or cyclic cracking of the cell walls. ${ }^{[1,2]}$

\section{CONCLUSIONS}

The heat generated upon localized cyclic deformation of a cellular Al results in a small temperature rise. The temperature distribution measured using an IR imaging system has been compared with that calculated using a model wherein heat is generated by the cyclic plasticity occurring within a Dugdale zone. The correlation indicates a consistency that reflects the utility of the Dugdale approach. The temperature elevations are not large enough to be responsible for fatigue failure.

\section{REFERENCES}

1. Y. Sugimura, A. Rabiei, A. Harte, N.A. Fleck, and A.G. Evans: Mater. Sci. Eng. A, 1999, vol. 269, p. 38.

2. A. Harte, N.A. Fleck, and M.F. Ashby: Acta Mater., 1999, vol. 47, p. 2511.

3. K.M. Flores and R.H. Dauskardt: J. Mater. Res., 1999, vol. 14, p. 638.

4. A.T. Zehner and A.J. Rosakis: J. Mech. Phys. Solids, 1991, vol. 39, p. 385.

5. S. Suresh: Fatigue of Materials, Cambridge University Press, Cambridge, United Kingdom, 1991.

6. M.N. Riddell, G.P. Koo, and J.L. O'Toole: Polymer Eng. Sci., 1967, vol. 6, p. 363.

7. Y. Sugimura, J. Meyer, M.Y. He, H. Bart-Smith, J. Grenestedt, and A.G. Evans: Acta Mater., 1997, vol. 45, p. 5245.

8. A. Simone and L.A. Gibson: Acta Mater., 1998, vol. 46, p. 2139.

9. A.G. Evans, J.W. Hutchinson, and M. F. Ashby: Progr. Mater. Sci., 1998, vol. 43, p. 171. 
10. V.S. Deshpande and N.A. Fleck: J. Mech. Phys. Solids, in press.

11. J.R. Rice: in Fracture, H. Liebowitz, ed., Academic Press, New York, NY, 1968, vol. II, p. 191.

12. H. Tada, P.C. Paris, and G.R Irwin: The Stress Analysis of Cracks
Handbook, ASME, New York, NY, in press.

13. M.F. Ashby, A.G. Evans, N.A. Fleck, L.J. Gibson, J.W. Hutchinson, and H.G. Wadley: Metal Foam Design Guide, Butterworth/Heineman, Stoneham, MA, in press. 